\title{
Radiation Response of Circulating SLAM Markers in Mice Treated with Papaya Leaf Extract and Diallyl Disulfide
}

\author{
Yogish Somayaji', Vidya Vasudeva ${ }^{2}$, Jayarama Shetty ${ }^{3}$, Guruprasad Parashiva ${ }^{4}$, Suchetha Kumari ${ }^{5}$ \\ 'Department of Post Graduate Studies and Research in Biochemistry, St. Aloysius College (Autonomous), Mangaluru, Karnataka, INDIA. \\ ${ }^{2}$ Central Research Laboratory, K S Hegde Medical Academy, Nitte University, Mangaluru, Karnataka, INDIA. \\ ${ }^{3}$ Department of Oncology, Nitte Leela Narayan Shetty Memorial Institute of Oncology, Mangaluru, Karnataka, INDIA. \\ ${ }^{4}$ Department of aging research, School of life sciences, Manipal University, Manipal, Udupi, Karnataka, INDIA. \\ ${ }^{5}$ Department of Biochemistry, K S Hegde Medical Academy, Nitte University, Mangaluru, Karnataka, INDIA.
}

\begin{abstract}
Background: CD48, CD150 and CD244.2 are the signaling lymphocytic activation molecules (SLAM) of hematopoietic population expressed on the $T$ cells, B cells, monocytes, macrophages and play an important role in adaptive immune response. Aim: The present study aims at evaluating the individual circulating levels of these SLAM markers in response to electron beam radiation and the protective effects of Carica papaya (L.) and DADS intervention. Methods and Material: The mice were fed orally with $100 \mathrm{mg} / \mathrm{kg}$ body weight of papaya leaf extract and $10 \mathrm{mg} / \mathrm{kg}$ body weight of DADS. The extract and DADS were administered to individual groups for 5 consecutive days with respective control groups and exposed to 6Gy of electrons radiation. Results: The present study indicated significant rise in the CD244.2 levels in irradiation control and a near normal levels in groups with C.papaya and DADS intervention. A significant decrease in the CD48 expression of granulocytes is seen in radiation control group on comparison to the normal control group. A significant rise is seen in the CD48 expression of granulocytes with DADS treatment prior irradiation when compared to DADS control. A significant increase in the granulocyte CD150 expression
\end{abstract}

with DADS treatment prior irradiation group is seen when compared to the DADS control group. In DADS treatment prior irradiation compared to radiation control the CD244.2 expression in granulocytes is significantly lowered. Conclusion: Study suggests a potential increase in the percentage of $\mathrm{CD} 244.2^{+}$lymphocytes in response to electron beam radiation and a comparative decrease in the percentage of CD244.2+ lymphocytes in C.papaya leaf extract and DADS intervention.

Key words: SLAM markers, Immune suppression, Hematopoietic progenitors, CD48, CD150, CD244.2, NK cell, Cytotoxicity.

\section{Correspondence}

Dr. Suchetha Kumari N, Department of Biochemistry, K S Hegde Medical Academy, Nitte University, Mangaluru, Karnataka, INDIA.

Phone: +919448451318

Email: kumari.suchetha@gmail.com

DOI: 10.5530/jyp.2019.11.45

\section{INTRODUCTION}

Total body irradiation (TBI) and its detrimental effect on the blood and bone marrow cause depletion of the bone marrow stem cells and progenitor cells leading to loss of hematopoietic and immune function. ${ }^{1}$ The signaling lymphocytic activation molecules (SLAM) help in the identification of hematopoietic stem cells and their subsequent subpopulations in the bone marrow. ${ }^{2}$ CD48, CD150 and CD244 are the SLAM markers of hematopoietic population expressed on the T cells, B cells, monocytes, macrophages and play an important role in adaptive immune response..$^{3-4} \mathrm{CD} 150$ along with CD48 associates with src homology 2- domain- containing tyrosine phosphatase (SHP-2) which is thought to be involved in signal transduction. ${ }^{3} \mathrm{CD} 48$ plays a role in adhesion and $\mathrm{T}$ cell recognition. ${ }^{3}$ CD244.2 belongs to the CD2 family of proteins expressed on all natural killer (NK) cells. ${ }^{5}$ CD244 along with CD48 is needed for the IL-2 driven expansion and activation of murine NK cells. ${ }^{6}$ Upon antibody mediated ligation or engagement by CD48, the CD244 gets recruited to the membrane microdomains at NK cell immunological synapse (NKIS) and the immunoreceptor tyrosine based switch motifs (ITSMs) become phosphorylated initiating a signaling cascade resulting in NK cell cytotoxicity and secretion of cytokines ${ }^{7-8}$ The individual expression of these markers could also provide information about cell signaling and inflammation in response to ionizing radiation. Carica papaya (L.) leaf extract has shown thrombopoietic activity in dengue infections. ${ }^{9}$ Also it has been proven to have anticlastogenic and antiproliferative effects in mice. ${ }^{10}$ Diallyl disulphide (DADS) is a component of garlic which has also been proven to have anti-cancer and anticlastogenic potential. ${ }^{11}$ In the present study individual circulating levels of SLAM markers in response to electron beam radiation and the protective effects of Carica papaya (L.) and DADS intervention is evaluated.

\section{MATERIALS AND METHODS}

The study was ethically approved by the institutional animal ethics committee of K S Hegde Medical Academy, Nitte University. Inbred male Swiss albino mice, 6-8 weeks old, weighing $25 \pm 5 \mathrm{~g}$ were used for the study.

FITC anti-mouse CD48 (Cat\#103403), PE anti-mouse CD150 (Cat\#115903) and PE-Cy7 anti-mouse CD244.2 (Cat\#133511) were purchased from Biolegend Inc. San Diego. Diallyl disulphide was purchased from TCI chemicals pvt. ltd., Japan. The aqueous leaf extract of papaya was prepared from the locally grown 'pusa' variety.

The mice were fed orally with $100 \mathrm{mg} / \mathrm{kg}$ body weight of papaya leaf extract and $10 \mathrm{mg} / \mathrm{kg}$ body weight of DADS. These doses were optimized for maximum survival at high radiation doses. The extract and DADS were administered to individual groups for 5 consecutive days with respective control groups. On the $5^{\text {th }}$ day, the mice were exposed to $6 \mathrm{~Gy}$ of electrons radiation at the Radiotherapy unit of NLNSM cancer hospital 
at a dose rate of $3 \mathrm{~Gy} / \mathrm{min}$ at a source to target distance of $100 \mathrm{~cm}$. The blood was collected within $24 \mathrm{~h}$ after irradiation and processed for flow cytometry analysis.

\section{Flow cytometry analysis}

Whole blood $(50 \mu \mathrm{L})$ was aliquated into different Falcon tubes and $10 \mu \mathrm{L}$ of specific antibody was added (as per the instruction of manufacturer) to different tubes, mixed thoroughly and incubated in dark for 30 mins at room temperature. Later, $1 \mathrm{~mL}$ of $\mathrm{BD}$ cell lysis buffer $(1 \mathrm{X})$ was added and kept for $15 \mathrm{mins}$ at room temperature. The samples were centrifuged for 5 mins at 3000rpm and the supernatant was discarded. The cell pellets were then suspended with $1 \mathrm{~mL}$ phosphate buffered saline (PBS) (BD; 1X) and centrifuged at 3000rpm for 5 mins. The supernatant was discarded and the cell pellets were re-suspended in $1 \mathrm{~mL}$ PBS and the samples were acquired in BD FACS Calibur flow cytometer. The data of 10,000 cells were recorded. The data of expression of SLAM markers on the mononuclear cell population and the granulocyte cell populations was analyzed using Cell Quest Pro software. The differences between the groups were determined using the statistical software PRISM 6.0.

\section{RESULTS}

The difference in percentage lymphocyte count and granulocyte count between different groups is shown in Figures $1(\mathrm{~A})$ and $1(\mathrm{~B})$. There is a significant decrease $(p<0.05)$ in the percentage of lymphocytes seen with radiation control (RC) when compared to the normal control group (NC) and between DADS treatment prior irradiation group (DR) when compared to DADS control group (DC). There is significant increase $(p<0.05)$ in the percent granulocytes in radiation control group $(\mathrm{RC})$ when compared to the control group. A significant increase $(p<0.01)$ in percent granulocyte count is seen with the DADS treatment prior irradiation group (DR) when compared to the DADS control group (DC).

The CD48 expression by granulocytes and lymphocytes with papaya extract and DADS intervention is shown in Figures $2(\mathrm{~A}), 2(\mathrm{~B}), 2(\mathrm{C})$ and 2(D). A significant decrease $(p<0.05)$ in the CD48 expression of granulocytes is seen in the radiation control group $(\mathrm{RC})$ in comparison to the normal control group $(\mathrm{NC})$. A significant rise $(p<0.01)$ is seen in the CD48 expression of the granulocytes with DADS treatment prior irradiation (DR) when compared to DADS control (DC).

The CD150 expressed by granulocyte and lymphocyte with papaya extract and DADS intervention is shown in Figures 3(A), 3(B), 3(C) and $3(D)$. A significant increase $(p<0.05)$ in the granulocyte CD150 expression with DADS treatment prior irradiation group (DR) is seen when compared to the DADS control (DC) group.

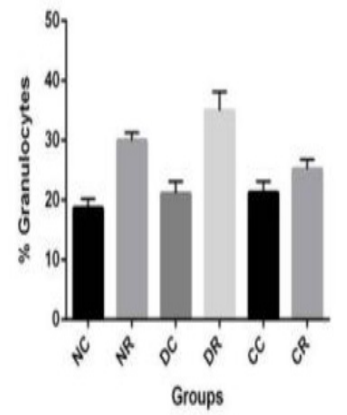

$1 \mathrm{~A}$

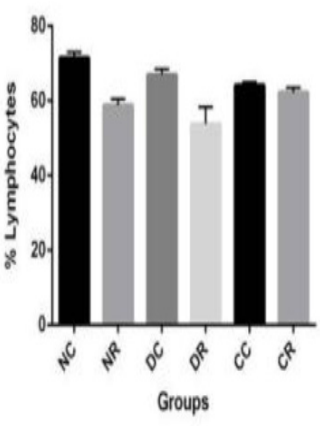

$1 B$

Figure $\mathbf{1 a}$ and $\mathbf{1 b}$ : Showing the percent granulocyte and lymphocyte count in untreated group $(\mathrm{NC})$, radiation control (NR), papaya extract control $(\mathrm{CC})$ and papaya treatment prior irradiation group (CR), DADS control (DC) and DADS treatment prior irradiation group (DR).
The granulocyte and lymphocyte expression of CD244.2 with papaya extract and DADS intervention is shown in Figures 4(A), 4(B), 4(C) and $4(\mathrm{D})$. In DADS treatment prior irradiation (DR) compared to radiation control (RC) the CD244.2 expression in granulocytes is significantly lowered $(p<0.05)$. The CD244.2 expression on the lymphocytes is increased significantly $(p<0.01)$ in radiation control (RC) and in C.papaya treatment prior irradiation (CR) group when compared to their respective control groups.

\section{DISCUSSION}

In the blood, the response to radiation is rapidly observed in cell count, under and over expression of certain cell specific immune function. ${ }^{11}$ Thus blood is the ideal target for radiation biodosimetry. The expression of specific cell type in response to radiation may activate the inflammatory pathway leading to immune suppression and apoptosis. A significant immune suppression was seen in non-small cell lung cancer that manifests decreased lymphocyte count and depression of natural killer activity
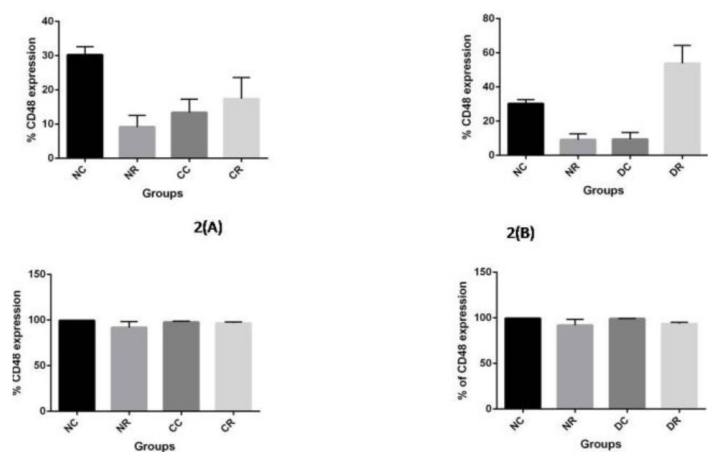

2(C)

2(D)

Figure 2: 2(A) showing the comparison of percent granulocyte CD48 expression between the groups; Figure 2(B) showing the comparison of percent lymphocyte CD48 expression; Figure 2(C) showing the comparison of percent lymphocyte CD48 expression; Figure 2(D)- showing the comparison of percent lymphocyte CD48 expression Untreated group (NC), radiation control (NR), papaya extract control (CC) and papaya treatment prior irradiation group (CR); DADS control (DC) and DADS treatment prior irradiation group (DR).

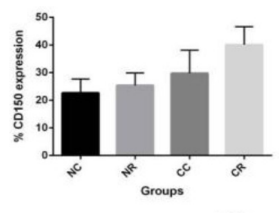

$3(\mathrm{~A})$

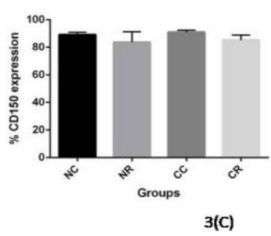

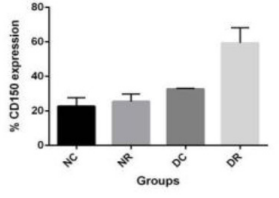

3(B)

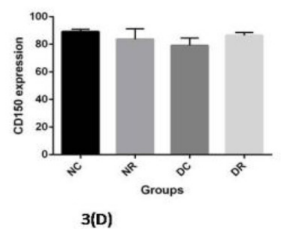

3(D)
Figure 3: 3(A) showing the comparison of percent granulocyte CD150 expression; Figure 3(B).jpg - showing the comparison of percent granulocyte CD150 expression; Figure $3(C)$ - showing the comparison of percent lymphocyte CD150 expression; Figure 3(D)- showing the comparison of percent lymphocyte CD150 expression; between the untreated group (NC), radiation control (NR), papaya extract control (CC) and papaya treatment prior irradiation group (CR); DADS control (DC) and DADS treatment prior irradiation group (DR). 

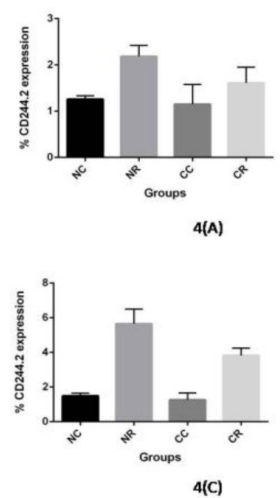

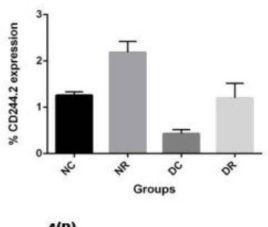

4(B)

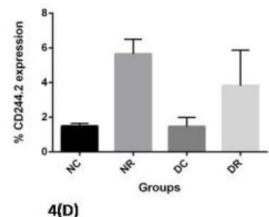

4(D)
Figure 4: 4(A).jpg - showing the comparison of percent granulocyte CD244.2 expression; Figure $4(B)$ - showing the comparison of percent granulocyte CD244.2 expression; Figure 4(C)- showing the comparison of percent lymphocyte CD244.2 expression; Figure 4(D)- showing the comparison of percent lymphocyte CD244.2 expression; untreated group (NC), radiation control (NR), papaya extract control (CC) and papaya treatment prior irradiation group (CR); DADS control (DC) and DADS treatment prior irradiation group (DR).

due to stereotactic beam radiotherapy. ${ }^{12}$ In the present study there was a significant decrease in the total WBC count, lymphocyte count seen in irradiated groups and subsequent normal levels were seen in C. papaya and DADS intervention. A transient increase in granulocytes seen in irradiated groups may be a counter effect of lymphopenia. C. papaya has already undergone extensive research in its platelet enhancing potential. ${ }^{913-14}$ DADS also has been known for its antitumor and antimicrobial activity. ${ }^{15-16}$

The CD150 expressed by the hematopoietic stem cells (HSCs), CD244.2 expressed by the transiently reconstituting multipotent progenitors (MPPs) and CD48 expressed by restricted progenitors (not HSCs or MPPs) were previously studied in the bone marrow with a SLAM code of $\mathrm{CD} 150^{+} \mathrm{CD} 244.2^{-\mathrm{CD}} 48^{-}$cells for hematopoietic progenitor cells (HPCs). ${ }^{3}$ The individual expression of these SLAM markers in circulation and their changes in response to radiation would be beneficial in understanding the hematological and immunological roles of these markers. The present study indicated significant rise in the lymphocyte CD244.2 levels in irradiation control and a near normal levels in groups with C. papaya and DADS intervention. There was a decrease in the granulocyte CD48 expression levels in the radiation control group when compared to normal control but this effect was normalized in DADS and C. papaya intervention prior irradiation. These results indicate that electron beam radiation induces changes in the lymphocyte CD244.2 and granulocyte CD48 levels which may be responsible for the radiation induced hematopoietic and immune suppression. The results also indicate immune stimulatory role of C. papaya and DADS in vivo. There were no significant differences seen in the granulocyte and lymphocyte CD150 expression levels between the intervention and control groups studied.

CD244.2 plays an important role in inflammatory response through its activating response to NK cells, interferon- $\gamma$ and tumor necrosis factor production which had yielded a negative effect on muscle fibre function and muscle performance in polymyositis and dermatomyositis patients. ${ }^{17}$ CD244.2 was shown to be a negative regulator of cytotoxic intra-epithelial $\mathrm{T}$ cells that regulate inflammation in the small intestine and cytokine production in NK cells. ${ }^{18}$ CD28 $8^{\text {null }}$ T cells express CD244.2 on their surface have been found to be enhanced in idiopathic inflammatory myopathies which are characterized by presence of lymphopenia, elevated proinflammatory $\mathrm{T}$ cell population and deficiency of suppressive regulatory $\mathrm{T}$ cell population. ${ }^{19} \mathrm{CD} 244.2$ alone plays an important role in inhibition of NK cell cytotoxic function which may otherwise lead to severe immune suppression by killing CD8 T cells. ${ }^{20}$ Immune cell populations respond differently depending upon which compartment they are in. ${ }^{21}$ In studies combining radiotherapy and immunotherapy, NK cells sensitize the tumor cells to radiation and the radiation sensitizes the tumor cells to NK cell attack. ${ }^{22}$ It was also observed that CD244.2 mediates NK/NKT-like cell activation with its mediated signaling contributing both positively and negatively to viral clearance and immune mediated diseases. ${ }^{23}$ Our results indicate a rise in the CD244.2 levels in radiation control group which may be the cause of immunological suppression seen in our previous studies. ${ }^{10}$ This could be attributed to its role as an NK cell activator which was reduced in response to C. papaya and DADS treatment prior irradiation. The C. papaya and DADS treatment prior irradiation has shown to reduced CD244.2 levels also correlates with the protective effects seen in the hematological and cytogenetic parameters studied previously. ${ }^{10}$

\section{CONCLUSION}

The present study suggests a potential increase in the percentage of CD244.2 $2^{+}$lymphocytes in response to electron beam radiation and a comparative decrease in the percentage of CD244.2+ lymphocytes in C.papaya leaf extract and DADS intervention. Thus, CD244.2+ could be a potential target for radiation biodosimetry and also a therapeutic target for radioprotectors. Also, the other SLAM markers in combination can be used to determine the HPC population in bone marrow and circulation with respect to different doses of ionizing radiation. CD244.2 and CD48 might play an important role in cell signaling and inflammation in response to ionizing radiation.

\section{ACKNOWLEDGEMENT}

The authors sincerely thank Prof. (Dr.) Sathyamoorthy, Director, School of Life Sciences, Manipal University, Manipal for his support in utilizing the flow cytometry lab. The authors also thank Dr. Mohana Kumar B, Prof. Dr. Palaksha KJ and Mrs. Jyothi for their support to carry out the work. The work was funded by board of research in nuclear sciences (BRNS) Department of atomic energy (DAE), government of India (Sanction No.2012/34/26/BRNS). The research work was carried out at the central research laboratory of K S Hegde medical laboratory, Nitte university, Mangaluru.

\section{CONFLICT OF INTEREST}

The authors declare no conflict of interest.

\section{ABBREVAITIONS}

DADS: Diallyl disulfide; SLAM: Signaling Lymphocyte Activation Molecule; SHP2: Src homology -2- domain- containing tyrosine phosphatase; NK: Natural Killer Cell; NKIS: NK Cell Immunological Synapse; ITSM: Immunoreceptor Tyrosine Based Switch Motifs; HPC: Hematopoietic progenitor cells; HSC: Hematopoietic Stem Cells; MPP: Multipotent progenitor cells.

\section{REFERENCES}

1. Shao $L$, LuoY, Zhou D. Hematopoietic stem cell injury induced by ionizing radiation. Antioxidants and redox signaling. 2014;20(9):1447-62.

2. Oguro H, Ding L, Morrison SJ. SLAM family markers resolve functionally distinct subpopulations of hematopoietic stem cells and multipotent progenitors. Cell Stem Cell. 2013;13(1):102-16.

3. Kiel MJ, Yilmaz OH, Iwashita T, Yilmaz OH, Terhorst C, Morrison SJ. SLAM family receptors distinguish hematopoietic stem and progenitor cells and reveal endothelial niches for stem cells. Cell. 2005;121(7):1109-21.

4. Ray A, Yuan CY, Miller NM, Mei H, Dittel BN. 2B4 Is Dispensable for T-Dependent B Cell Immune Responses, but Its Deficiency Leads to Enhanced T-Independent Responses Due to an Increase in Peritoneal Cavity B1b Cells. PLoS One. 2015;10(8):e0137314.

5. Claus M, Wingert S, Watzl C. Modulation of natural killer cell functions by inter- 
actions between 2B4 and CD48 in cis and in trans. Open Biol. 2016;6(5):160010.

6. Alari-Pahissa E, Grandclément C, Jeevan-Raj B, Leclercq G, Viellette A, Held W. Activation by SLAM Family Receptors Contributes to NK Cell Mediated "Missing-Self" Recognition. PLoS One. 2016;11(4):e0153236.

7. G"utgemann SA, Mina MS, Wingert S, Claus M, Watzl C. Recruitment of activating NK-cell receptors 2B4 and NKG2D to membrane microdomains in mammalian cells is dependent on their transmembrane regions. Eur $\mathrm{J}$ Immunol. 2015;45(4):1258-69.

8. Carsten W, Maren C. WhatSAP-2B4 sends mixed messages in the absence of SAP. Eur J Immunol. 2014;44(5):1281-4

9. Dharmarathna SLCA, Wickramasinghe S, Waduge RN, Rajapakse RPVJ, Kularatne SAM. Does Carica papaya leaf-extract increase the platelet count? An experimental study in a murine model. Asian Pac J Trop Biomed. 2013;3(9):720-4.

10. Yogish ST, Vidya V, Shama R, Damodara G, Chandrika R, Ganesh S, et al. Hematopoietic, antioxidant and membrane stabilizing property of diallyl disulphide in irradiated mice. J Cli Diag Res. 2016;10(2):BF01-BF05.

11. Paul S, Smilenov LB, Amundson SA. Widespread decreased expression of immune function genes in human peripheral blood following radiation exposure. Radiat Res. 2013;180(6):575-83.

12. Maehata $Y$, Onishi $H$, Kuriyama $K$, Aoki $S$, Araya M, Saito R, et al. Immune responses following stereotactic body radiotherapy for stage I primary lung cancer. BioMed Res Intl. 2013.

13. Subenthiran S, Choon TC, Cheong KC, Thayan R, Teck MB, Muniandy PK, et al. Carica papaya leaves juice significantly accelerates the rate of increase in platelet count among patients with dengue fever and dengue haemorrhagic fever. $J$ Evid Based Complementary Altern Med. 2013.

14. Gammulle A, Ratnasooriya WD, Jayakody JRAC, Fernando C, Kanatiwela C Udagama PV. Thrombocytosis and anti-inflammatory properties and toxicological evaluation of Carica papaya mature leaf concentrate in a murine model. Int J Med Plant Res. 2012;1(2):21-30
15. Reddy BS, Rao CV, Rivenson A, Kelloff G. Chemoprevention of colon carcinogenesis by organosulfur compounds. Cancer Res. 1993;53(15):3493-8.

16. Robert V, Mouille B, Mayeur C, Michaud M, Blachier F. Effects of the garlic compound diallyl disulfide on the metabolism, adherence and cell cycle of HT-29 colon carcinoma cells: evidence of sensitive and resistant sub-populations. Carcinogenesis. 2001;22(8):1155-61.

17. Pandya JM, Ingela $\mathrm{L}$, Mohammad $\mathrm{SH}$, Alexanderson $\mathrm{H}$, Raghavan $\mathrm{H}$, Lundberg IE, et al. Effects of conventional immunosuppressive treatment on CD244+ (CD28null) and FOXP3 + T cells in the inflamed muscle of patients with polymyositis and dermatomyositis. Arthritis Res Ther. 2016;18(1):80.

18. Michael SO, Joo-Hye S, Gongxian L, De Calisto J, Halibozek PJ, Mora JR, Bhan AK, et al. SLAMF4 is a negative regulator of expansion of cytotoxic intra-epithelial CD8+ T cells that maintains homeostasis in the small Intestine. Gastroenterol. 2015;148(5):991-1001.

19. Espinosa-Ortega F, Gómez-Martin D, Santana-De AK, Romo-Tena J, Villasenor-Ovies P, Alcocer-Varela J. Quantitative T cell subsets profile in peripheral blood from patients with idiopathic inflammatory myopathies: tilting the balance towards proinflammatory and pro-apoptotic subsets. Clin Exp Immunol. 2014;179(3):520-8

20. Stephen NW, Vinay K. Evolving role of 2B4/CD244 in T and NK cell responses during virus infection. Front Immunol. 2012;3:337.

21. Jung E, Perrone EE, Brahmamdan P, McDounough JS, Leathersich AM, Dominguez JA, et al. Inhibition of intestinal epithelial apoptosis improves survival in a murine model of radiation combined injury. PLoS One. 2013;8(10):e77203.

22. Yang KL, Wang YS, Chang CC, Huang SC, Huang YC, Chi MS, et al. Reciprocal Complementation of the Tumoricidal Effects of Radiation and Natural Killer Cells. PLoS One. 2013;8(4):e61797.

23. Thanapati S, Das R, Tripathy AS. Phenotypic and functional analyses of NK and NKT-like populations during the early stages of chikungunya infection. Front Microbiol. 2015;6:895

Article History: Submission Date : 11-07-2018; Revised Date : 15-08-2018; Acceptance Date : 15-11-2018.

Cite this article: Somayaji YT, Vidya V, Shetty J, Guruprasad KP, Kumari SN. Radiation Response of Circulating SLAM Markers in Mice Treated with Papaya Leaf Extract and Diallyl Disulfide. J Young Pharm. 2019;11(2):220-3. 